\title{
Analysis on Emotional Design of Daily Ceramic Products
}

\author{
Dehua $\mathrm{Yu}$ \\ The Department of Art and Design \\ Hunan City University \\ Yiyang, Hunan, China, 413000
}

\begin{abstract}
Ceramic products are widely used in daily life, and not out of service even with the appearance of cheap plastic products and expensive metalware. The reasons are their wild texture and centuries-old cultural inheritance. At present, the design of daily ceramic products follows cultural pattern and emotional literacy of traditional ceramic products, highly combines emotional literacy with using function, giving consumers emotional experiences on the basic of meeting the necessary need. The importance of emotional factors is increasingly obvious, because it is the demonstration of property attribute and decided by its characteristics. This paper will analyze the vital role of emotional literacy in ceramic products design from shape expression, form language, material aesthetics and design ideas by analyzing emotional factors of visual image, interactive experience and cultural patterns of daily ceramic products.
\end{abstract}

Keywords-emotional factors; form; design language; interactive experiences

\section{INTRODUCTION}

Functions and aesthetics are indispensable in daily ceramic products. If we simply regard the ceramic products as common industrial products, and overlook the cultural connotation and aesthetic consciousness, the design of ceramic products will lost too much. Designers should regard servqual empathy of users in the using process of daily ceramic products as a fatal factor, because it is the brand that connects functions and aesthetics and determines the effect of the products and experiences of users. A good daily ceramic product should not only function as bearing, storage and aesthetics, but also should make users happy and express their feelings. It should be culture carriers and emotional sustenance. Only fully considering emotional needs and psychological experiences of users, highlighting its aesthetics characteristics and interactive attribute by shape, structure, color, material and texture of products, can we really touch the users and produce a positive influence.

\section{DESIGN REPRESENTATION OF DAILY CERAMIC PRODUCTS}

Design of daily ceramic products is represented by structure, color, decoration and material to reach expected function of usage and aesthetics. Representing cultural connotation by their quality, texture, design, structure, culture and environment and giving users complicated emotional experiences by visual effects of smooth, mildness and humanistic beauty. However, with the innovation of concept of product development, consumers change their demands of products soon, so the products must be intellectualized, individualized, bionics and interesting to give users multiple experiences to arouse the enthusiasm of consumers, establish consuming expectations and produce surprise and attraction to make consumers active respond to products. Design skills such as symbolization, image and connection should be used to motivate good imagination of users and putting emotional factors into shape, color, design, material, texture and hierarchical structure, relieving products with environment to produce deep and complete experiences. For example, putting connective design factors into certain daily ceramic products and designing different styles for different consuming groups.

\section{A. Design of Shape.}

Shape design of daily ceramic products isn't independent, it should be regarded as an organism that connects users and environment, it also should relate to living space, individual experiences and culture background of that time. Ceramic products can be divided into daily ceramic products and decorative ceramic products according to their functional characteristics. The biggest characteristic of daily ceramic products in shape design is to fully consider contact experiences of users, and this is what collectors said "appreciation". Tea set used to drink tea and coffee set used to drink coffee have the same function and shape, but they are different in appreciation mentality, so it is necessary to infer emotional psychology of users in shape design. Daily ceramic products are various in shape, their different shapes give users different emotional experiences. Bionics type and mimicry type aim at emphasizing visual beauty of objects themselves in nature and expressing a happy, natural and harmonious using experience at same time. They exist in space shape, the exaggerated virtual space design gives a person the visual aesthetic feeling of emptiness and broadminded psychological experience, and the pragmatic virtual space conveys static aesthetic feeling and inclusive psychological experience. 


\section{B. Utilization of Materials.}

Daily ceramic products are mainly composed of kaolin, mineral firing glaze layer, their material characteristic are bright and clean and beautiful, high hardness, crisp and plastic, etc. the smooth and meticulous quality gives a person exquisite, delicate, light, health, comfortable feelings and emotional experiences. In the design of daily ceramic products, designers can make full use of the advantages of material, get better access to consumer identity, through delicate and mild touch on them to convey the spiritual characteristics of the daily ceramic products in household, better complement with environment and bring out the best in each other. Such as the design of the coffee set, the designer can combine the body language with psychological characteristics of young group, using the plasticity of the ceramic material in a personalized form language to give users dynamic, diverse and rich emotional experiences by the products. Designers can also use crisp sound of ceramic to create ceramic products with acoustical characteristic for users to create a more multiple-experience.

\section{Expression of Decoration.}

Decorative expression methods and forms of daily ceramic products are various, such as traditional pattern design, modern graphic decoration and personalized design, etc. Decoration expression relates to colors, combines pictures with colors to give products a specific character and give users corresponding emotional experiences. We should take view of value, cultural background and life style and customs of consumers into consideration when designing so that the users can be happy and using the products with appreciation. Decorative colors are based on the shape of products and reflect emotional features of them. In practical design, suitable color contrast makes decorative effect of products more vivid. Decoration is also the carrier of culture, it is added value of functions and aesthetics of products and connected factors to appreciate and use products "Fig. 1".

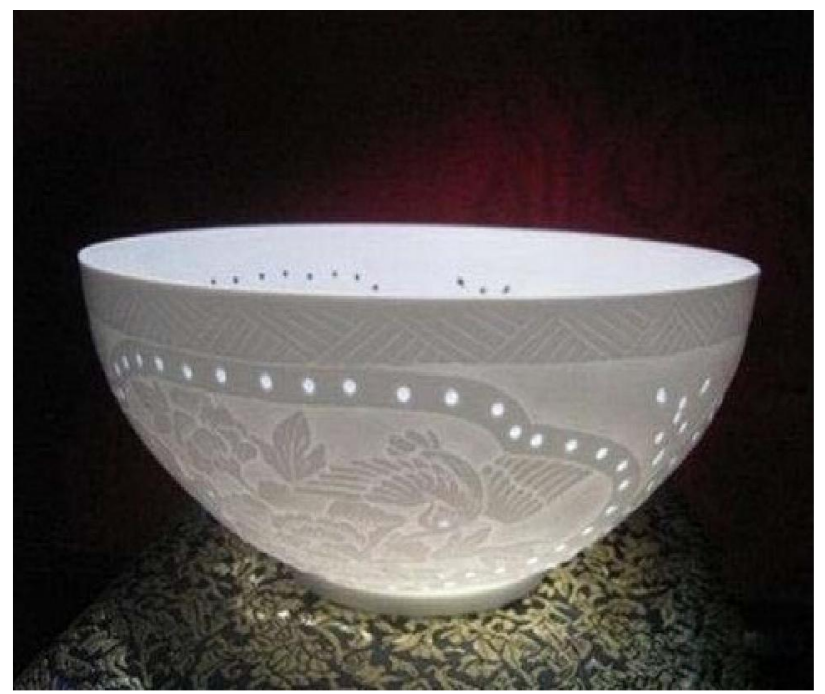

Fig. 1. Decorative expression of daily ceramic products

\section{Design of DAILy CERAMIC PRODUCTS}

Design of daily ceramic products can be connected by three levels of correlation: instinct correlation, behavior correlation and reflection correlation. Designing visual appearance of daily ceramic products according to instinct correlation and creating direct and visible images of products with visual signs. Give users interactive communication experiences in the process of utilization by behavior correlation, with speciality of ceramics and characters of human, regarding products design as the organic combination of people and environment. Valuate symbolic meaning and cultural orientation by reflection correlation to integrate view of value, aesthetic ideal and spiritual needs of users together to produce products with high art aesthetics.

\section{A. Instinct Correlation and Design of Daily Ceramic Products}

Instinct correlation of daily ceramic products are mainly focused on the appearance of products, it expresses direct feeling of first impression. It is composed of profile, structure, space, color and decoration of products and is also the first level that designers pay attention to in design. Only touch users on the design of appearance can products get more development space. Restricted by functions, the appearances of daily ceramic products are limited by and large. For example, tea pot consists of pot body, pot handle or girder, lid and button, spout, or flow, etc. Pot body can be divided into the neck, shoulder, abdomen, waist and foot as well as the proportion, cohesion, angle, curvature of all parts, etc. According to the principle of ergonomics, it has reached a relatively perfect form. But under the creative genius of crafts the designer, each pot presents a different mental state and a different emotion. Take the girder flat pot "inner peace" designed by Han Meilin and Gu Jingzhou for example, with a round body and extravagant girder, the whole shape both real and virtual, giving a sedate and deft visual perception to people. Highly integrating the functional requirements of teapot that are commonly used in the life with aesthetics embodies the ethereal and elegant interest of tea, makes people enjoy people calm and comfortable spirit "Fig. 2".

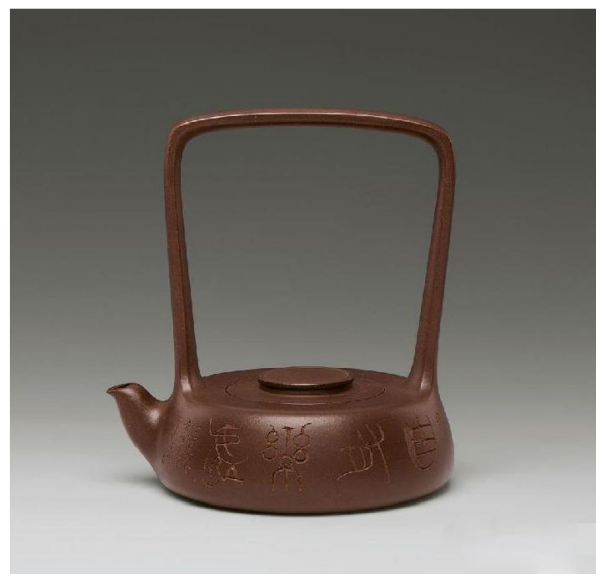

Fig. 2. The girder flat pot "inner peace" designed by Han Meilin and Gu Jingzhou 


\section{B. Behavior Correlation and Design of Daily Ceramic Products}

Behavior correlation is a key part in daily ceramic products design and emotional factors are adequately emphasized and displayed in it. In the interactive process of users and products, users will experience the pleasure of participation and contact well. At present, many products add interesting factors into appearance design such as ricepattern decorated porcelain in Jingde town. This kind of products is dexterous, clear, translucent, unsophisticated and fresh. Further design on its clearness and combination of light and environments, the users can experience the pleasure better and get good interactive experiences between people and products. The design of daily ceramic products also concerns the temperature difference between users and objects, this humanized design method makes people interact with products by body language.

\section{Reflection Correlation and Design of Daily Ceramic Products}

Reflection correlation is embodied in products consideration of cultural patterns, spiritual needs and social values in the design of daily ceramic products. It requires designers fully understand ceramic products' cultural ecology, current context and product symbolic as well as their signifier and referent. At present, the design of daily ceramic products pays more attention to their deeper level factors such as symbolic meanings, symbol value and emotional appeal, the need of internal emotions like interest and entertainment is also gradually become one design conception. Daily ceramic products is a part of both life and culture, it is influenced by culture and influences the existence of culture as well, just as the culture and living habits of different regions influence different emotional appeal and appearance of different products. "When we choose and evaluate a product, emotion can be a key factor in the process. Consumers express different emotions to different products according to their own characters and preference, at the same time, and these complicated emotions determine behaviors of consumers"

\section{CONCLUSION}

This is an experience economy age, so consumers value emotional pleasure and satisfaction more at the same time they value functions and quality. The emotional consuming demand is the manifestation of human high-level requirements, and it reflects that modern people pay more attention to the satisfaction of spirit, emotion and characters. The integration of emotional factors influences the direction of daily ceramic products design. A product that touches people from its appearance contains emotional communication between designer, products and users in every detail. In fact, the shape, color and material of product design itself are combinations of real objects and invisible emotional factors, and it has the characters that touch people. What designers should do is to motivate emotional factors of products, make the aesthetics and practicability of products highly unified, take full advantage of the influence of emotional factors to make the products more humanized and of more social value.

\section{REFERENCES}

[1] Yin Dingbang. Introduction to Design[M].Hunan: Hunan Science and Technology Press,2004, 10

[2] Donald A. Norman. Emotionalized Design[M]. Beijing:Electronic Industry Press,2005

[3] Norman Denson. Emotionalist Theory--translated by Wei Zhongjun[M].Liaoning: Liaoning People's Publishing House, 1989, 68-70

[4] Jin Wenwei. the Life Feelings of Ceramic[J]:Deccoration:2006, 10 